\title{
Reducing Fraudulent News Proliferation using Classification Techniques
}

\author{
A Raghuvira Pratap, Prasad J V D, Sallagundla Babu, V V N V Phani Kumar
}

\begin{abstract}
The expansion of dishonorable information in normal get entry to social access media retailers like internet based media channels, news web journals, and online papers have made it hard to identify dependable news sources, subsequently growing the need for technique tools able to deliver insights into the reliability of online content substances.. This paper comes up with the applications of Natural language process techniques for detective work the dishonest news, that is, dishonorable news stories that return from the non-reputable sources. Solely by building a model supported mistreatment word tallies or a Term Frequency-Inverse Document Frequency matrix, will solely get you to date. Is it potential for you to make a model which will differentiate between "Real "news and "Fake" news? Thus our planned work is going to be on grouping a knowledge set of each pretend and real news and uses a Nä̈ve mathematician classifier so as to make a model to classify an editorial into pretend or really supported its words and phrases.
\end{abstract}

Index Terms: Fraudulent, Fake News, Natural language processing, TF-IDF, Fake News.

\section{INTRODUCTION}

Fake news is never again an original thought. Significantly, the idea has been in presence strikingly sooner than the development of the Internet as publishers utilized fake and deceptive data too comparably with their inclinations. Following the making of the web, an ever increasing number of consumers began neglecting the standard media channels used to scatter statistics for on-line stages. Not exclusively does the last decision grant clients to get admission to a scope of publications in a single sitting, anyway it is additionally extra comfort and quicker.The advancement, be that as it may, accompanied a re-imagined thought of fake news as substance publishers started the utilization of what has come to be normally alluded to as click bait. Clickbait's are phrases that are intended to entice the consideration of an individual who, after tapping on the connection, is coordinated to a web page whose content is definitely beneath their desires.

Revised Manuscript Received on February 05, 2020.

* Correspondence Author

A Raghuvira Pratap*, Department of Computer Science and Engineering, V.R.Siddhartha Engineering College,Andhra Pradesh, Vijayawada, India. raghuvirapratap@gmail.com

Prasad J V D , Department of Computer Science and Engineering, V.R.Siddhartha Engineering College,

Andhra Pradesh, Vijayawada, India. prasadjasti2018@gmail.com

Sallagundla Babu, Department of Computer Science and Engineering, V.R.Siddhartha Engineering College,Andhra Pradesh, Vijayawada, India. babunaidu.504@gmail.com

V V N V Phani Kumar, Department of Computer Science and Engineering, V.R.Siddhartha Engineering College,Andhra Pradesh, Vijayawada, India. phanikumar.venna@gmail.com,

(C) The Authors. Published by Blue Eyes Intelligence Engineering and Sciences Publication (BEIESP). This is an open access article under the CC BY-NC-ND license (http://creativecommons.org/licenses/by-nc-nd/4.0/)
Numerous users find misleading baits sources to be a bothering, and the final product is that the majority of such people exclusively wind up investing an exceptionally short energy venturing such locales. As a creating proportion of our lives is spent working together on-line through web based systems administration stages, a regularly expanding number of people tend to are looking out and eat information from Internet based media as a substitute than ordinary news affiliations.

The reasons behind this other in use practices are intrinsic in the possibility of these web based life channels; it is a significant part of the time progressively noticeable advantageous and less expensive to gobble up information by means of online systems administration media differentiated and standard news media, for instance, news papers or TV; and it is less difficult to moreover share, remark on, and talk about the news with partners or distinctive per clients by means of electronic systems administration media.

The extensive spread out of artificial news can have a genuinely horrible impact on individuals and society. In the first place, fake information can break the validity reliability of the information organic framework. For example, it is evident that the most notable phony information was impressively more comprehensively spread on Face book than the most acclaimed veritable standard information at some stage in the U.S. 2016 president decisions. Second, counterfeit information purposely persuades clients to take transport of uneven or double dealings. Fake news is regularly constrained by promoters to pass on political messages or effect.Best case scenario, tech organizations, for example, Google, Face book, and Twitter have attempted to address this exact concern. Nonetheless, these endeavors have seldom contributed nearer to taking care of the issue as the organizations have turned to denying the people related with such sites the pay that they would have acknowledged from the duplicated traffic.Customers, at the one of a kind hand, continue to cope with sites containing false records and whose involvement tends to have an effect on the reader's potential to interact with right information.

\section{RELATED WORK}

Mykhailo Granik et.al [1] presents method "titled as Fake News Detection using Naïve Bayes Classifier". This strategy was once applied as a software program device and examined towards records set of Face book news posts. And they performed classification; these results might also be expanded in several approaches that are described in the paper as well. 
Shivam B. Parikh et.al [2] introduced a new methodology titled as "Media-Rich Fake news detection: A survey". They talk about the sorts of information that the reports are made of, there are 4 significant configurations they are content, sound, interactive media, hyperlinks, in which clients expend their news. Right now, hop into existing phony news disclosure approaches toward that are strongly established on content based assessment, and besides delineate popular phony news educational assortments.

Kai Shu et.al [3] defined a technique titled as "Understanding User Profiles on Social Media for Fake News Detection". Right now, on fake news and select agent gatherings of both "experienced" clients who can perceive fake news things as false and "gullible" users who are bound to accept counterfeit news. Points of interest are It gives to comprehend the relationship between client profiles and phony news, Disadvantages are fake news deliberately deludes individuals to accept fake data and change the manner in which individuals react to genuine news.

Shaban Shabani et.al [4] proposed a method titled as "Hybrid Machine-Crowd Approach for Fake News Detection". At this moment the human factor with the AI approach and a fundamental dynamic model that checks the grouping conviction of estimations and picks whether the task needs human data or not. Our methodology accomplishes sensibly higher exactness contrasted with the detailed benchmark results, in return of cost and idleness of utilizing the publicly supporting assistance.

Shlok Gilda et.al [5] defined a method titled as "Evaluating Machine Learning Algorithms for Fake News Detection". In this they use different machine learning algorithms and they also discuss performance of the algorithms respectively.

\section{METHODOLOGY}

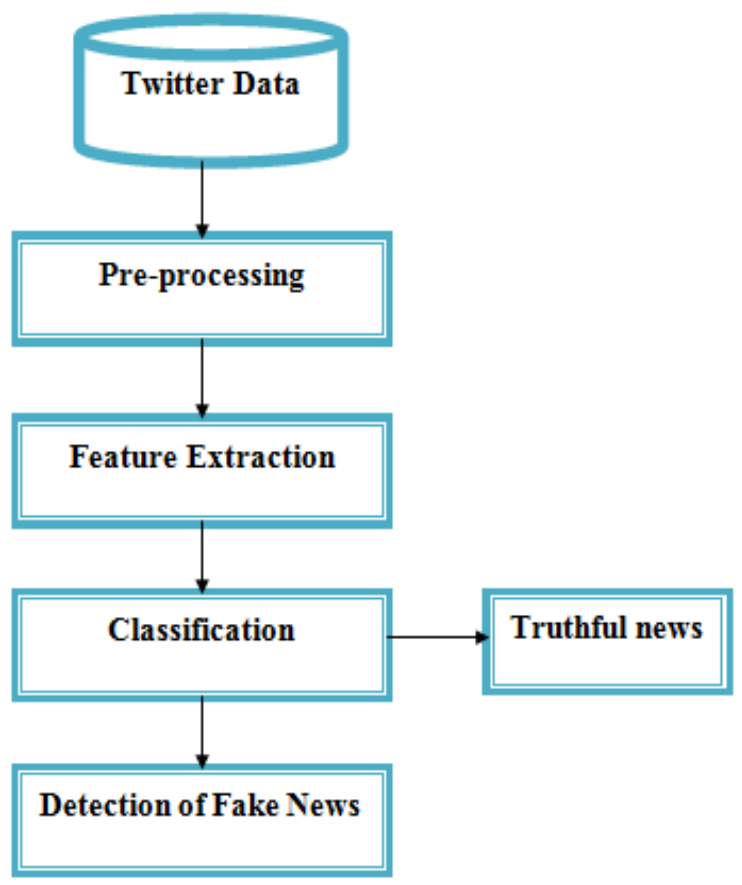

“Fig 1.Proposed Methodology"

\section{A. Data Set}

A dataset was taken from a kaggle-based websites. And the data set contains text data; it is in the form of .csv format. In this, data set consists of number of statements along with labels are true or false respectively.

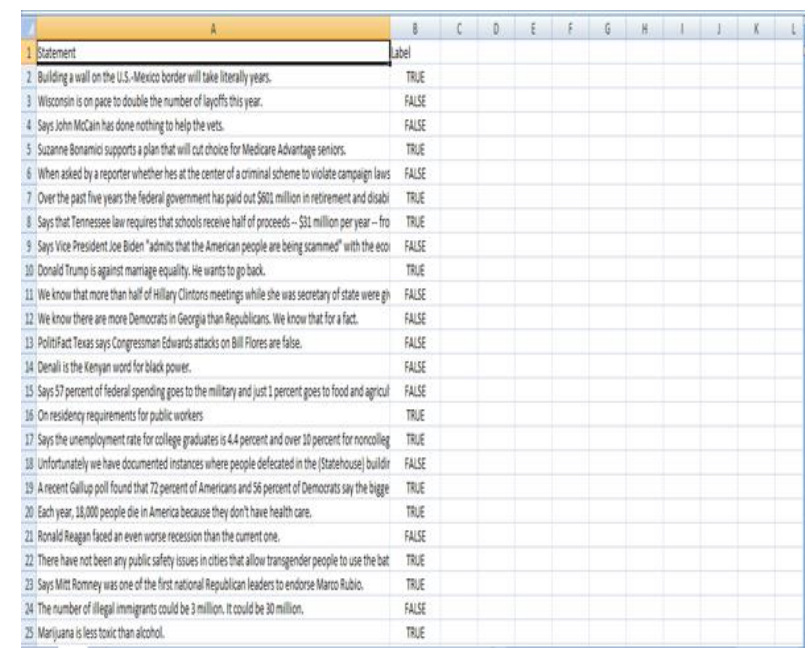

"Fig 2. Twitter Data Set"

\section{B. Pre-Processing}

In this, pre-handled and to dispose of undesirable information like Stop words, copied words, clear spaces and so on. Before addressing the information using n-gram and vector-based model, the data ought to be presented to certain clarification like stop-word clearing, tokenization, a lower bundling, sentence division, and highlight ejection. This will help us with diminishing the size of real information by removing the irrelevant data that exists in the information.

\section{Feature Extraction}

Text categorization is gaining from high dimensional information. There are an enormous assortment of terms, words, and expressions in records that cause a high technique trouble for the preparation procedure. In addition, insignificant and repetitive features can hurt the precision and execution of the classifiers. In this manner, it's ideal to perform feature decrease to scale back the text element estimate and maintain a strategic distance from monster include area measurement. In this, we have a tendency to study during this analysis two totally different options choice strategies, to mention, Term Frequency and Term Frequency-Inverted Document Frequency. N-gram features are removed, and a features grid is framed representing to the documents involved.

\section{Classification}

After feature extraction stage classification was acted right now. Classification begins with pre-processing the informational index, by expelling superfluous characters and words from the data. The last advance in the characterization procedure is to prepare the classifier.

We investigated different classifiers to predict the class of the documents. We researched various classifiers to foresee the class of the documents. 
We explored explicitly two distinctive machine learning calculations, to be specific,

1. Support Vector Machines (SVM)

2. Random Forest Classifier

We utilized usage of these classifiers from the Python Natural Language Toolkit.

\section{E. Detection of Fake News}

After, classification was done, we automatically discover the news is fake or not individually. Machine learning classification algorithms are utilized to find detection of fake news from twitter data. And also calculates the accuracy of twitter data using classification algorithms.

\section{F. Prediction}

Our finally picked and best performing classifier was calculation which was then saved money on plate with name file_modal.sav. At the point when you close this store, his model will be replicated to customer's machine and will be used by predict.py archive to arrange the fake news with accuracy. It takes a news story as contribution from client at that point model is utilized for conclusive characterization yield that is appeared to client alongside likelihood of truth.

\section{RESULTS}

The model was implemented using python and NLTK tool kit. The accuracy results as shown in below table and visualization done in graph. As shown in table 1, Accuracy of around $99.25 \%$ while training and for validation the model give around $95 \%$ in both models.

"Table 1: Shows proposed model and comparison model accuracy results"

\begin{tabular}{|c|c|}
\hline Model & Accuracy \\
\hline SVM & 83.74 \\
\hline Random Forest & 92.45 \\
\hline
\end{tabular}

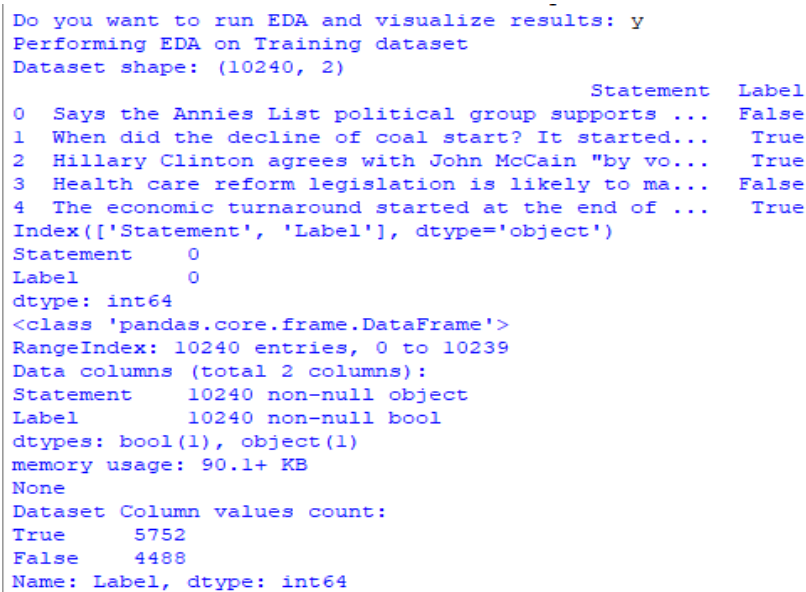

"Fig3. EDA Visualize results in pre-processing"

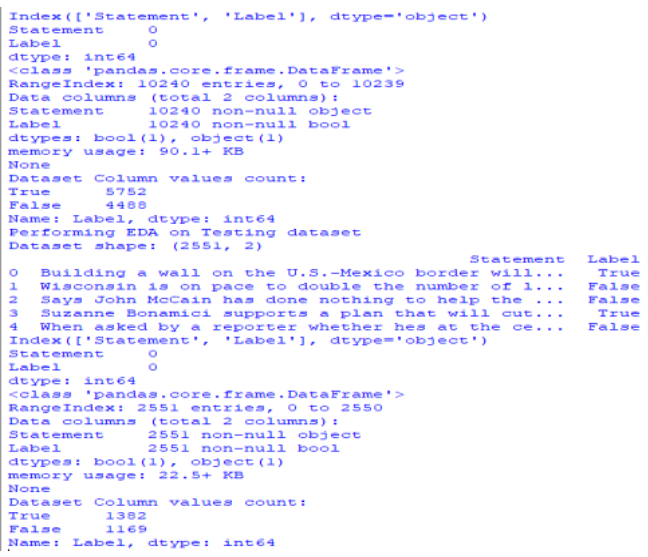

"Fig 3.EDA Performance"

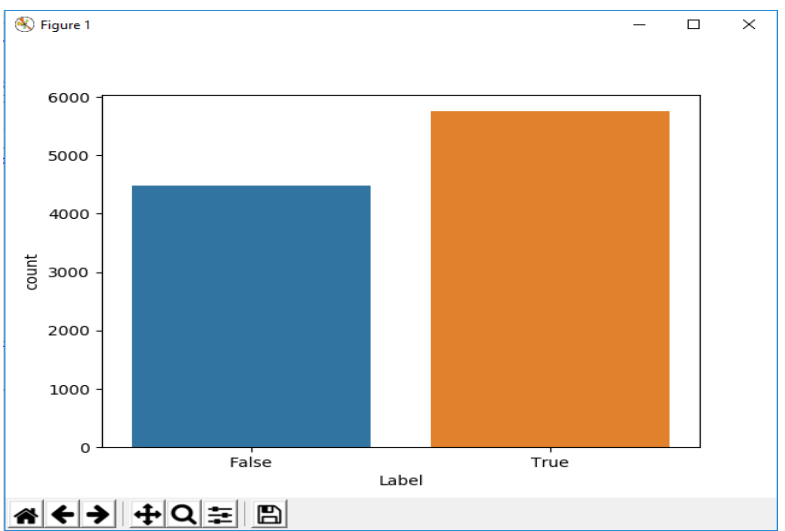

"Fig 4.Output of Random Forest Classification"

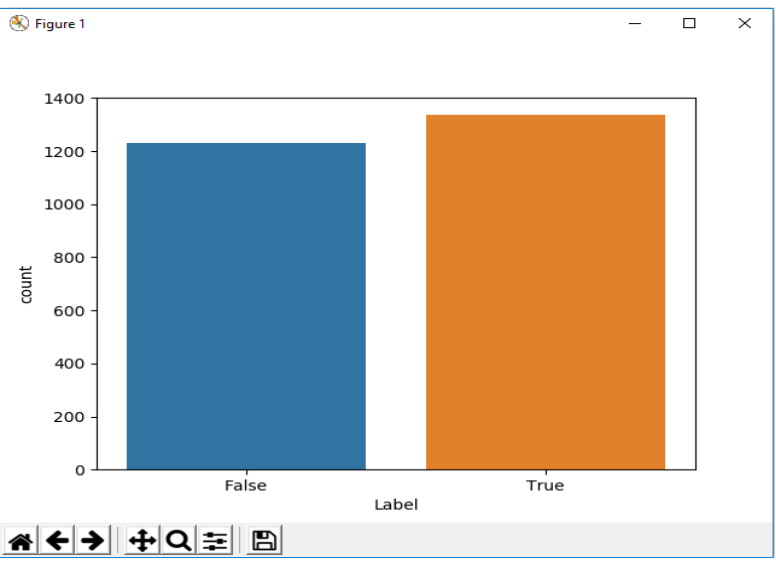

"Fig 5.Output of SVM Classification"

\section{CONCLUSION}

In this article, we have proposed another system for fake news location on twitter data. fake news identification is accustomed to distinguishing individuals' feeling, mentality and enthusiastic states. The perspectives on the individuals can be sure or negative. Usually, parts of speech are utilized as feature to remove the sentiment of the text. Examination on around 100 tweets is performed. So we investigate the results, comprehend the patterns and offer a survey on individual's opinions. It isn't important that the classifier must be utilized for a particular theme. It is general classifier. It tends to be utilized for any reason dependent on tweets we gather with the assistance of keyword.

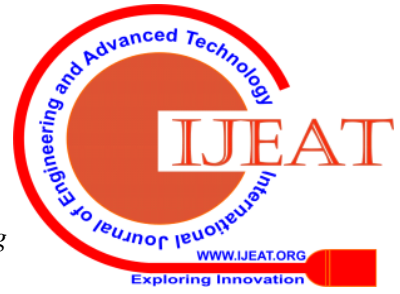




\section{REFERENCES}

1. Granik M, Mesyura V. Fake news detection using naive Bayes classifier. InElectrical and Computer Engineering (UKRCON), 2017 IEEE First Ukraine Conference on 2017 May 29 (pp. 900-903). IEEE.

2. Macro L,E Tacchini, S Moret, G Ballarin, "Automatic Online Fake news Detection combining content and social signals".

3. Parikh SB, Atrey PK. Media-Rich Fake News Detection: A Survey. In2018 IEEE Conference on Multimedia Information Processing and Retrieval (MIPR) 2018 Apr 10 (pp. 436-441). IEEE.

4. Shu K, Wang S, Liu H. Understanding user profiles on social media for fake news detection. In2018 IEEE Conference on Multimedia Information Processing and Retrieval (MIPR) 2018 Apr 10 (pp. 430-435). IEEE.

5. Shabani S, Sokhn M. Hybrid Machine-Crowd Approach for Fake News Detection. In2018 IEEE 4th International Conference on Collaboration and Internet Computing (CIC) 2018 Oct 18 (pp. 299-306). IEEE.

6. Gilda S. Evaluating machine learning algorithms for fake news detection. InResearch and Development (SCOReD), 2017 IEEE 15th Student Conference on 2017 Dec 13 (pp. 110-115). IEEE.

7. Buntain C, Golbeck J. Automatically Identifying Fake News in Popular Twitter Threads. In Smart Cloud (Smart Cloud), 2017 IEEE International Conference on 2017 Nov 3 (pp. 208-215). IEEE.

8. Mykhailo Granik, Volodymyr Mesyura, Andrii Yarovyi, "Determining Fake Statements Made by Public Figures by Means of Artificial Intelligence", Computer Sciences and Information Technologies (CSIT) 2018 IEEE 13th International Scientific and Technical Conference on, vol. 1, pp. 424-427, 2018.

\section{AUTHORS PROFILE}

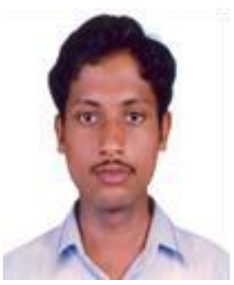

A RaghuViraPratap has received the $B$. Tech degree in Computer Science and Engineering from $\mathrm{V} \quad \mathrm{R}$ Siddhartha Engineering College,Vijayawada,India.He has received the $\mathrm{M}$. Tech degree in Computer Science and Engineering from PVP Siddhartha Institute of Technology, Vijayawada, India. His research interests include Web Technologies and Data Mining.He has over more than 12 years of teaching experience.Currently he is working as Assistant Professor in Computer Science and Engineering at VR Siddhartha Engineering College,Vijayawada,India.

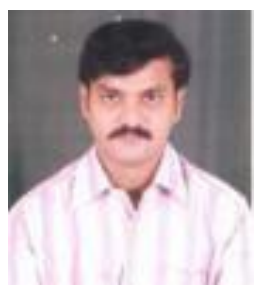

J V D Prasad has received the $M$. Tech degree in Computer Science and Engineering from V R Siddhartha Engineering College, Vijayawada, India. He is currently pursuing Ph.D. from Acharya Nagarjuna University His research interests include Data Mining and Parallel Computing. He has over more than 15 years of teaching experience. Currently he is working as Assistant Professor in Computer Science and Engineering at VR Siddhartha Engineering College, Vijayawada, India

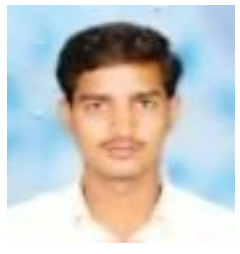

Babu Sallagundla has received the B. Tech degree in Computer Science and Engineering from Priyadarsini College of Engineering, Sulurupet, India. He has received the $\mathrm{M}$. Tech degree in Computer Science and Engineering from $\mathrm{V} R$ Siddhartha Engineering College,

Vijayawada, India. His research interests include Web Technologies and Data Mining. He has over more than 11 years of teaching experience. Currently he is working as Assistant Professor in Computer Science and Engineering at VR Siddhartha Engineering College, Vijayawada, India.

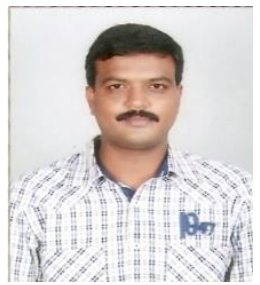

V V N V Phani Kumar has received the B. Tech degree in Information Technology from PVP Siddhartha Institute of Technology, Vijayawada, India. He has received the $M$. Tech degree in Computer Science and Engineering from VR Siddhartha Engineering College, Vijayawada, India. His research interests include Cloud computing and Data Mining.He has over more than 11 years of teaching experience.Currently he is working as Assistant Professor in Computer Science and Engineering at VR Siddhartha Engineering College, Vijayawada,India. 